\title{
Proteoglycan Form of Macrophage Colony-stimulating Factor Binds Low Density Lipoprotein
}

\author{
Shinya Suzu, *ll Toshimori Inaba, * Nobuya Yanai, * Takuji Kawashima," Nobuhiro Yamada, ${ }^{*}$ Teruaki Oka, ${ }^{5}$ \\ Rikuo Machinami, s Tetsuya Ohtsuki," Fumihiko Kimura," Shuichi Kondo,' Chikao Torikata, ' Naokazu Nagata," \\ and Kazuo Motoyoshil \\ ${ }^{*}$ The Biochemical Research Laboratory, Morinaga Milk Industry Co., Ltd., Kanagawa 228; ${ }^{\ddagger}$ The Third Department of Internal Medicine \\ and ${ }^{8}$ The Department of Pathology, Faculty of Medicine, University of Tokyo, Tokyo 113; and "The Third Department of Internal \\ Medicine and 'The Department of Pathology, National Defense Medical College, Saitama 359, Japan
}

\begin{abstract}
We recently isolated a proteoglycan form of macrophage colony-stimulating factor (PG-M-CSF) that carries a chondroitin sulfate glycosaminoglycan chain. Here, we examined the interaction of PG-M-CSF with low density lipoprotein (LDL). When LDL preincubated with PG-M-CSF was fractionated by molecular size sieving chromatography, it was eluted earlier than untreated LDL. When LDL was preincubated with chondroitin sulfate-free 85-kD M-CSF instead of PG-M-CSF, the elution profile of LDL remained unchanged, indicating specific interaction between PG-M-CSF and LDL. The level of PG-M-CSF binding in the wells of a plastic microtitration plate precoated with LDL was significant, this binding being completely abolished by pretreatment of PG-M-CSF with chondroitinase $\mathrm{AC}$, which degrades chondroitin sulfate. The addition of exogeneous chondroitin sulfate or apolipoprotein $B$ inhibited the binding of PG-M-CSF to LDL in a dose-dependent manner, indicating that the interaction between PG-M-CSF and LDL was mediated by the binding of the chondroitin sulfate chain of PG-M-CSF to LDL apolipoprotein B. PG-M-CSF was also demonstrated in the arterial wall, and there were increased amounts of PG-M-CSF in atherosclerotic lesions. The in vitro interaction between PG-M-CSF and LDL thus appears to have physiological significance. (J. Clin. Invest. 1994. 94:1637-1641.) Key words: colony-stimulating factor - chondroitin sulfate - glycosaminoglycan - atherosclerosis - macrophage
\end{abstract}

\section{Introduction}

Proteoglycans (PG) ${ }^{1}$ are macromolecules composed of various kinds of glycosaminoglycan (GAG) chains and various kinds of core proteins, whose moieties are covalently bound with each

Address correspondence to Kazuo Motoyoshi, M.D., The Third Department of Internal Medicine, National Defense Medical College, Namiki 3-2, Tokorozawa, Saitama 359, Japan.

Received for publication 13 October 1993 and in revised form 1 June 1994.

1. Abbreviations used in this paper: GAG, glycosaminoglycan; M-CSF, macrophage colony-stimulating factor; PG, proteoglycan.

\section{J. Clin. Invest.}

(C) The American Society for Clinical Investigation, Inc.

0021-9738/94/10/1637/05 \$2.00

Volume 94, October 1994, 1637-1641 other $(1,2)$. Common GAG include heparan sulfate, chondroitin sulfate, dermatan sulfate, and keratan sulfate $(1,2)$, the core proteins of these being different. The PG are a highly diverse group of macromolecules; in only a limited number has the molecular structure been established $(1,2)$.

PG are widely distributed in animal tissues. The increased amounts of certain arterial PG in atherosclerotic aortas and their affinity for low density lipoprotein (LDL) have lead various investigators to suggest that these PG are involved in the atherosclerotic process (3-7). Supporting evidence for the involvement of the PG-LDL complex in the atherosclerotic process has been provided by the isolation of this complex from human vascular lesions (8). Interaction between $P G$ and $L D L$ is thought to be mediated by the binding of anionic GAG chains to the positively charged amino acid of apolipoprotein B (37). However, previous studies have also indicated that both core proteins and GAG are essential for the formation of these complexes and that the potential of PG to interact with LDL depends on the nature of the core proteins and on the compositions of the GAG $(4,8,9)$. The interaction between PG molecules and LDL is important in the pathogenesis of atherosclerosis, since this interaction may facilitate the trapping of LDL in the arterial wall or cause structural alterations in the LDL protein, alterations that influence the uptake of lipoprotein by the arterial mesenchyma (10-16). To date, however, no specific PG molecule involved in LDL binding has been characterized yet. To clarify the precise role of PG in the pathogenesis of atherosclerosis, it is important to identify PG molecules with such functions.

Macrophage colony-stimulating factor (M-CSF), a growth factor for mononuclear phagocytic cells (17), was first identified as a glycoprotein with a molecular mass of $85 \mathrm{kD}$ (18, 19). A second M-CSF molecule, with a molecular mass of $>200 \mathrm{kD}$, and which carries chondroitin sulfate GAG, has since been identified by us and others $(20,21)$; this was designated "proteoglycan form of M-CSF" (PG-M-CSF) (22). Human M-CSF is produced as a 522-amino acid polypeptide preceded by a 32 -amino acid signal peptide (19). The precursor polypeptides are rapidly dimerized via disulfide bonds and translationally glycosylated (19). Proteolytic cleavage at the residue around 223 produces an M-CSF subunit of $43 \mathrm{kD}$ (19). The $85-\mathrm{kD}$ M-CSF is a homodimer of the $43-\mathrm{kD}$ subunit (19). In contrast, proteolytic cleavage occurring at the residue around 400 , but not at that around 223, produces a PG-M-CSF-specific subunit with a molecular mass of $150-200 \mathrm{kD}(20,21)$. In addition to having a longer carboxyl terminus than the $43-\mathrm{kD}$ subunit, the PG-M-CSF-specific subunit contains a chondroitin sulfate chain on the $\mathrm{COOH}$-terminal portion (21). It has also been shown that the combination of the PG-M-CSF-specific 
subunit with the identical subunit or the 43-kD subunit lacking the $\mathrm{COOH}$-terminal structures yielded homodimeric or heterodimeric PG-M-CSF, respectively (22). Here, we show the capacity of this newly identified PG, i.e., PG-M-CSF, to bind plasma LDL.

\section{Methods}

Materials. The 85-kD M-CSF and PG-M-CSF were purified from media conditioned with Chinese hamster ovary cells transfected with human M-CSF cDNA $(19,21,22)$. Human LDL $(d=1.019-1.063 \mathrm{~g} / \mathrm{ml})$ was prepared by ultracentrifugation from normal plasma. Chondroitin sulfate A (whale cartilage), chondroitin sulfate B (hog skin), chondroitin sulfate C (shark cartilage), and heparan sulfate (bovine kidney) were purchased from Seikagaku Kogyo Co., Ltd. (Tokyo, Japan); purified human apolipoprotein B was purchased from Chemicon International, Inc. (Temecula, CA).

Formation of PG-M-CSF and LDL complex. Two types of associations between PG-M-CSF and LDL were explored.

First, soluble complexes were obtained by mixing solutions of LDL with solutions of M-CSF. LDL ( $100 \mu \mathrm{g}$ protein), 85-kD M-CSF (100 $\mu \mathrm{g}$ ), or PG-M-CSF ( $100 \mu \mathrm{g}$ protein) was dissolved in $1 \mathrm{ml}$ of phosphate-buffered saline (PBS). LDL ( $100 \mu \mathrm{g})$ was mixed with $85-\mathrm{kD} \mathrm{M}$ CSF $(100 \mu \mathrm{g})$ or PG-M-CSF $(100 \mu \mathrm{g})$ and dissolved in $1 \mathrm{ml}$ of PBS. These solutions were left at $37^{\circ} \mathrm{C}$ for $2 \mathrm{~h}$. A $10-\mu$ l aliquot of each solution was loaded on a TSK G3000SW column (Toyo Soda, Tokyo, Japan) and then eluted with $10 \mathrm{mM}$ Tris-HCl buffer ( $\mathrm{pH} 7.4$ ) containing $150 \mathrm{mM} \mathrm{NaCl}$. Fractions ( $400 \mu \mathrm{l} /$ fraction) were monitored for absorbance at $280 \mathrm{~nm}$ (23) and assayed for M-CSF content using an MCSF-specific enzyme-linked immunosorbent assay (ELISA) $(22,24)$.

Second, the binding of PG-M-CSF to immobilized LDL was examined. LDL ( $10 \mu \mathrm{g}$ protein $/ \mathrm{ml}$ in PBS) was added to the wells $(0.1 \mathrm{ml} /$ well) of a microtiter plate (3950; Costar Corp., Cambridge, MA), and the plate was incubated for $1 \mathrm{~h}$ at $37^{\circ} \mathrm{C}(7,22)$. The wells were then washed three times with PBS. All washing steps described below were done in the same manner. After the washing, the uncoated sites of the wells were blocked by incubation with $3 \%$ bovine serum albumin (BSA) in PBS for $90 \mathrm{~min}$. After further washing, samples dissolved in interaction buffer ( $10 \mathrm{mM}$ Tris- $\mathrm{HCl}$ buffer, $\mathrm{pH} \mathrm{7.0,} \mathrm{containing} 1.5 \% \mathrm{BSA}$ and $10 \mathrm{mM} \mathrm{CaCl}_{2}$ ) were then added to the coated wells, and incubation was carried out for $1 \mathrm{~h}$ at $37^{\circ} \mathrm{C}$. We included $\mathrm{CaCl}_{2}(10 \mathrm{mM})$ in the interaction buffer, since it had been reported in an earlier study that optimal binding between PG and LDL was obtained in the presence of divalent cations (7). In some experiments, PG-M-CSF was pretreated with chondroitinase AC ( $10 \mathrm{mU} / \mathrm{ml}$, EC 4.2.2.4; Seikagaku Kogyo Co., Ltd.) (12, 13) and then added to the wells, as outlined above. Potent inhibitors such as GAGs, soluble LDL, or apolipoprotein B were added to the PG-M-CSF solution immediately before the mixture was added to the wells. To keep apolipoprotein B soluble, we included sodium deoxycholate in the interaction buffer, at a final concentration of $10 \mathrm{mM}$, to the mixture of apolipoprotein B and PG-M-CSF, according to the manufacturer's instructions. The wells were then washed, anti-M-CSF rabbit IgG dissolved in PBS containing $1.5 \%$ BSA was added, and the plate was further incubated for $1 \mathrm{~h}$ at $37^{\circ} \mathrm{C}$. The plate was then washed, horseradish peroxidase-conjugated goat IgG against rabbit IgG (BioRad Laboratories, Richmond, CA), dissolved in PBS containing $1.5 \%$ BSA, was added, and the plate was incubated for another $1 \mathrm{~h}$ at $37^{\circ} \mathrm{C}$. After a final washing, freshly prepared $O$-phenylenediamine dihydrochloride solution was added, and the color development of the solutions in the wells was measured with absorbance at $492 \mathrm{~nm}$, using a microplate reader (Bio-Rad Laboratories).

Characterization of arterial M-CSF. The purified M-CSF preparations ( $1 \mu \mathrm{g}$ each) were dialyzed against buffer A ( $25 \mathrm{mM}$ Tris- $\mathrm{HCl}$, $\mathrm{pH} 7.4$, containing $0.5 \%$ CHAPS and $6 \mathrm{M}$ urea) and then applied to a 1-ml DEAE-Sephacel (Pharmacia LKB Biotechnology Inc., Piscataway, $\mathrm{NJ})$ column previously equilibrated with buffer $\mathrm{A}$. The column was washed stepwisely with $2 \mathrm{ml}$ of buffer $\mathrm{A}$, containing $0.1,0.2,0.3,0.4$,
$0.5,0.6,0.7$, or $0.8 \mathrm{M} \mathrm{NaCl}$. The fractions, at a $1: 1,000$ dilution, were then assayed for M-CSF content $(22,23)$. The purified M-CSF preparations were also analyzed by gradient (4-20\% acrylamide) SDS-PAGE under nonreduced conditions, followed by immunoblotting $(21,24)$. Aortas were obtained at autopsy from five patients with atherosclerosis, after family members had given their informed consent. The intimal layers of the normal and atherosclerotic regions were peeled off and weighed. $10 \mathrm{ml}$ of ice-cold extraction buffer $(25 \mathrm{mM}$ Tris- $\mathrm{HCl}$ buffer, pH 7.4, containing $6 \mathrm{M}$ urea, $1 \mathrm{M} \mathrm{NaCl}, 0.5 \%$ CHAPS, $10 \mathrm{mM}$ EDTA, $1 \mathrm{mM}$ phenylmethylsulfonyl fluoride, $5 \mathrm{mM}$ benzamidine- $\mathrm{HCl}$, and 10 $\mathrm{mM}$ aminocaproic acid) was poured over $1 \mathrm{~g}$ of the tissues. The tissues were then finely minced with scissors and extracted for $24 \mathrm{~h}$ at $4^{\circ} \mathrm{C}$. After centrifugation, the supernatants (tissue extracts) were collected. Each extract or normal serum $(1 \mathrm{ml})$ was dialyzed against buffer A, loaded onto a 2.5-ml DEAE-Sephacel column, and then eluted stepwisely with $5 \mathrm{ml}$ buffer, as outlined above. The fractions, at a 1:10 dilution, were then assayed for M-CSF content with an ELISA.

\section{Results}

If PG-M-CSF and LDL formed a complex, it would be expected that this complex would have a greater molecular size than noncomplexed LDL. To confirm this, we compared the elution profiles of LDL and LDL preincubated with PG-M-CSF in PBS on a TSK G3000SW column (Toso, Tokyo, Japan). Taking advantage of the nature of lipoproteins, i.e., their relatively high value of absorbance at $280 \mathrm{~nm}$ (23), we analyzed each fraction for LDL content by measuring absorbance at $280 \mathrm{~nm}$. As shown in Fig. $1 a, \mathrm{LDL}$ preincubated with PG-M-CSF was eluted in the inclusive volume of the column, with an elution time earlier than that of untreated LDL, indicating the formation of a complex between PG-M-CSF and LDL. Acetylated LDL preincubated with PG-M-CSF was also eluted earlier than untreated acetylated LDL (data not shown). In contrast, LDL preincubated with $85-\mathrm{kD}$ M-CSF had the same elution profile as that of untreated LDL (Fig. $1 a$ ). Each fraction was also assayed for M-CSF content by ELISA (Fig. $1 b$ ). LDL-treated 85-kD M-CSF had the same elution position as the $85-\mathrm{kD}$ M-CSF, indicating no specific interaction between $85-\mathrm{kD} \mathrm{M}-\mathrm{CSF}$ and LDL. On the other hand, PG-M-CSF mixed with LDL was eluted as a broad peak with a later elution time than the untreated PG-M-CSF.

We next examined, using a modified ELISA $(22,24)$, the binding of PG-M-CSF to LDL previously adsorbed onto the wells of a plastic microtitration plate. There was no significant binding of PG-M-CSF to wells not coated with LDL (data not shown), and there was no significant binding of the $85-\mathrm{kD} \mathrm{M}$ CSF to wells coated with LDL, while PG-M-CSF exhibited an appreciable capacity to bind LDL (Fig. $2 a$ ). Pretreating PG-MCSF with chondroitinase $\mathrm{AC}$, which degrades the chondroitin sulfate chains of PG-M-CSF, completely abolished the binding (Fig. $2 a$ ). When added to the interaction mixture, various GAG species, including three chondroitin sulfate preparations, inhibited PG-M-CSF binding to the immobilized LDL (Fig. 2 $b$ ). Heparan sulfate also inhibited the binding, but its inhibition was less effective than that of chondroitin sulfate GAG (Fig. 2 $b$ ). As shown in Fig. $2 c$, the interaction of PG-M-CSF with immobilized LDL was partly inhibited by the addition of soluble LDL; apolipoprotein B, a protein present in LDL, also exhibited efficient inhibitory activity. Taken together, these findings indicate that the interaction between PG-M-CSF and LDL was mediated by the binding of PG-M-CSF chondroitin sulfate to LDL apolipoprotein B. 

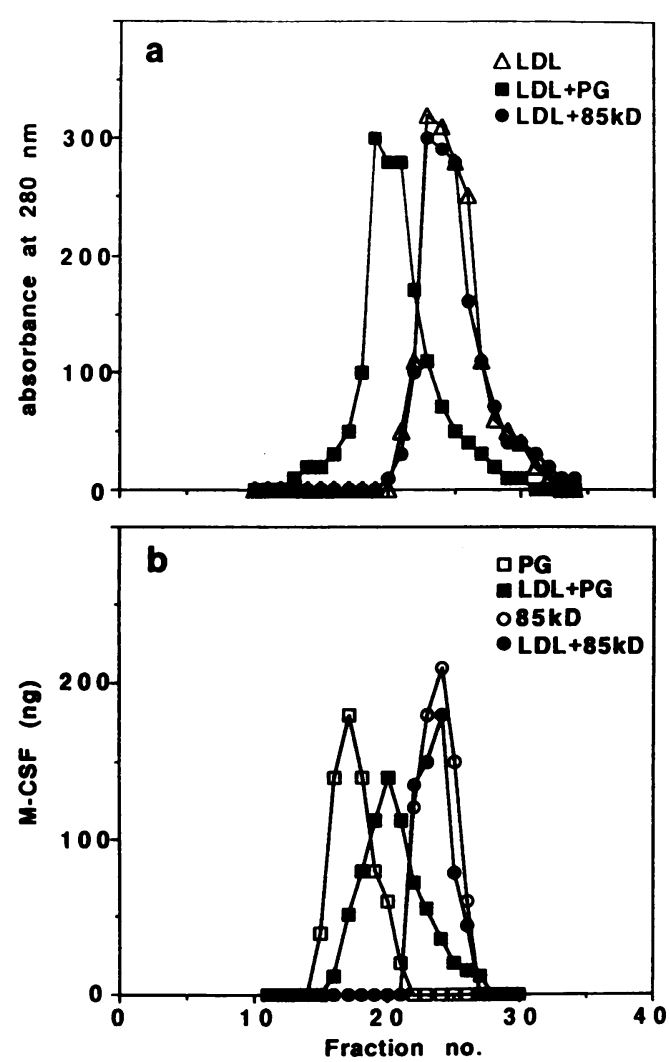

Figure 1. Chromatography of LDL and LDL preincubated with M-CSF on a TSK G3000SW column. LDL, 85-kD M-CSF ( $85 k D)$, PG-M-CSF $(P G)$, LDL preincubated with $85-\mathrm{kD}$ M-CSF $(L D L+85 k D)$, or LDL preincubated with PG-M-CSF $(L D L+P G)$ were eluted on a TSK G3000SW column. The eluted fractions were monitored for absorbance at $280 \mathrm{~nm}(a)$ or assayed for M-CSF content using an ELISA $(b)$. No detectable peak was observed when 85-kD M-CSF and PG-M-CSF fractions were monitored for absorbance at $280 \mathrm{~nm}$, and no significant amounts of M-CSF were detected when LDL fractions were assayed with an M-CSF-specific ELISA. The void-volume fraction is No. 13.

Our hypothesis, that PG-M-CSF may bind LDL in the arterial wall in vivo, was supported by the detection of PG-M-CSF in the arterial wall (Fig. 3). The 85-kD M-CSF and PG-MCSF showed different behavior on DEAE-Sephacel ion-exchange column chromatography (20-22). As shown in Fig. 3 $a$, the purified $85-\mathrm{kD}$ M-CSF (inset) was eluted mainly at 0.2 $\mathrm{M} \mathrm{NaCl}$ concentration, while the purified PG-M-CSF (inset) was eluted mainly at $0.4 \mathrm{M} \mathrm{NaCl}$ concentration. Using this system, we characterized the M-CSF species present in normal human serum and aortic extracts. The majority of serum MCSF was eluted at $0.2 \mathrm{M} \mathrm{NaCl}$, and there was a faint appearance of M-CSF at $0.4 \mathrm{M} \mathrm{NaCl}$ (Fig. $3 b$ ), indicating that the $85-\mathrm{kD}$ M-CSF was the major M-CSF species in human serum. The extracts of normal aortic tissue contained M-CSF at a concentration of $1.66 \pm 0.42 \mathrm{ng} / \mathrm{g}$ tissue $(n=5)$, and the extracts of atherosclerotic intima contained higher amounts of M-CSF $(4.04 \pm 1.14 \mathrm{ng} / \mathrm{g}$ tissue, $n=5)$ than those from the normal region. The difference of the M-CSF amount between normal region and atherosclerotic region was statistically significant at the level of $P<0.002$ (Student's $t$ test). The M-CSF species in the extract of normal aorta was eluted at $0.2 \mathrm{M} \mathrm{NaCl}$, and there was also a second peak observed at $0.4 \mathrm{M} \mathrm{NaCl}$, with a
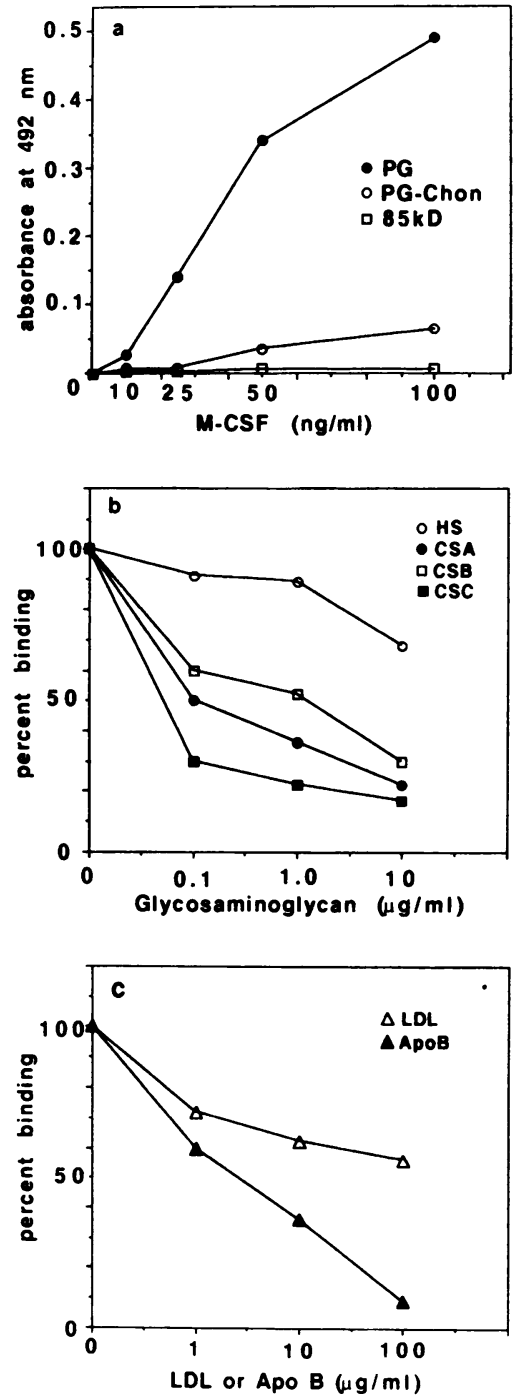

Figure 2. Binding of PGM-CSF to immobilized LDL. Purified 85-kD MCSF ( $85 k D)$, PG-MCSF $(P G)$, and PG-MCSF pretreated with chondroitinase AC ( $P G$ Chon) were diluted at the indicated concentrations and added to microtiter wells previously coated with LDL (a). PG-MCSF was mixed with heparan sulfate $(H S)$, chondroitin sulfate $A$ (CSA), chondroitin sulfate $\mathrm{B}(C S B)$, or chondroitin sulfate C (CSC) (b), or with LDL or apolipoprotein B (ApoB) (c). In the case of the mixture of apolipoprotein B and PG-M-CSF, sodium deoxycholate was included in the solution at a final concentration of $10 \mathrm{mM}$, as described in Methods. The presence of sodium deoxycholate at the concentration had no effect on PG-M-CSF binding to immobilized LDL. In both $b$ and $c$, the final concentration of PG-MCSF is $50 \mathrm{ng} / \mathrm{ml}$ and that of the GAG, LDL, or apolipoprotein B is indicated on the abscissa. Mixtures were added to wells coated with LDL, and the plate was pro-

cessed as indicated in $a$. The level of binding in the presence of a potential inhibitor was compared with the control binding obtained in its absence and was expressed as a percentage of the control binding. The PG-M-CSF samples without the potent inhibitors in $b$ and $c$ showed absorbances of 0.35 and 0.55 , respectively.

trailing shoulder at $0.5 \mathrm{M} \mathrm{NaCl}$ (Fig. $3 c$ ). There was no significant change in the M-CSF elution pattern of atherosclerotic intimal extract compared with that of the normal aorta, but both peaks contained approximately twofold larger amounts of $\mathrm{M}$ CSF than those in the normal aorta (Fig. $3 d$ ). The first peak at $0.2 \mathrm{M} \mathrm{NaCl}$ corresponding to 85-kD M-CSF of atherosclerotic region contained $0.77 \pm 0.32 \mathrm{ng} \mathrm{M}-\mathrm{CSF}$, which was higher than that of normal region $(0.43 \pm 0.10 \mathrm{ng})$ with a statistical significance at the level of $P<0.05$. Similarly, the second peak at $0.4 \mathrm{M} \mathrm{NaCl}$ corresponding to PG-M-CSF of atherosclerotic region contained $0.66 \pm 0.16 \mathrm{ng} \mathrm{M}-\mathrm{CSF}$, which was higher than that of normal region $(0.34 \pm 0.08 \mathrm{ng})$ with a statistical significance at the level of $P<0.004$. These findings indicated that both $85-\mathrm{kD}$ M-CSF and PG-M-CSF were present in the arterial wall and that both types of M-CSF were increased in atherosclerotic lesions. 

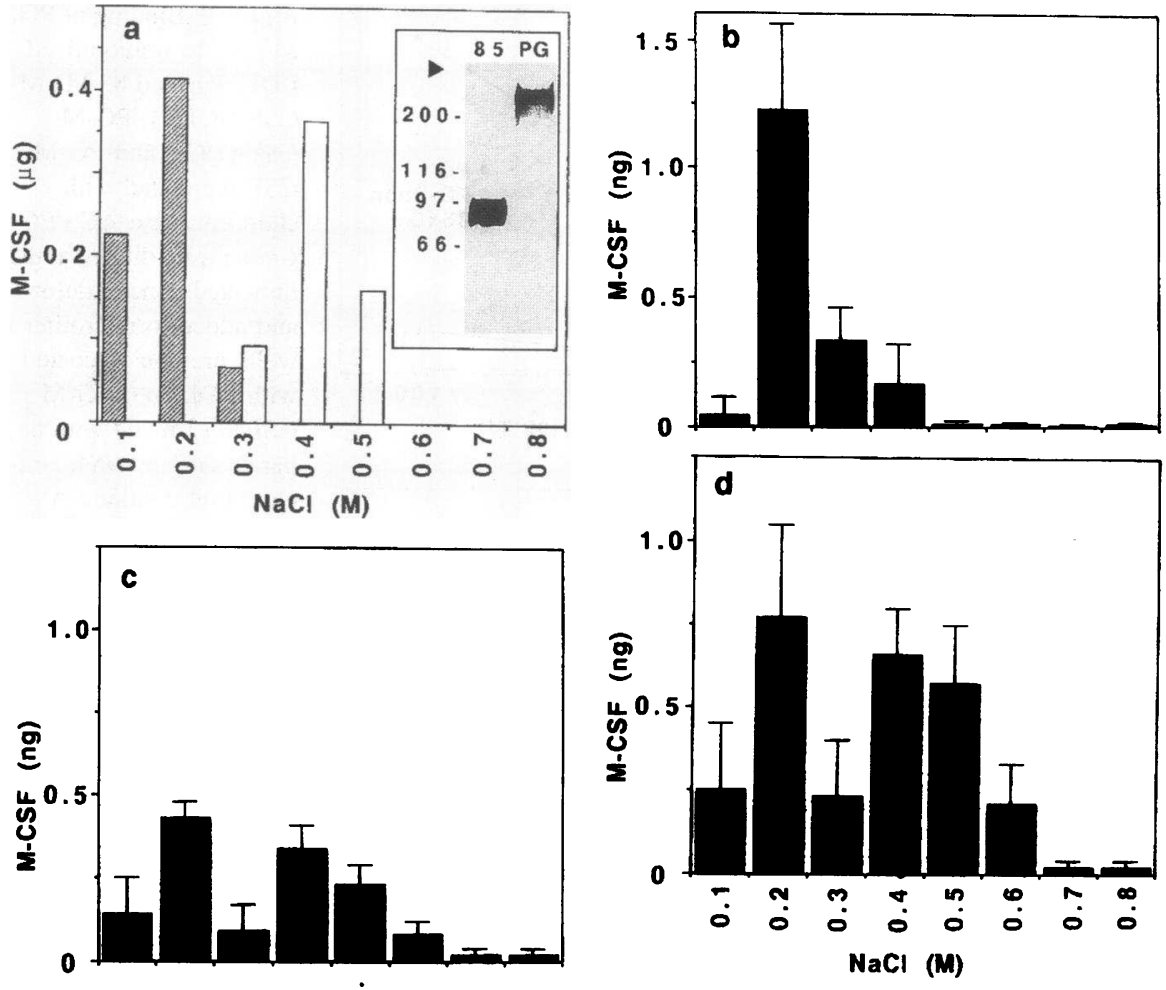

Figure 3. Chromatography of purified MCSFs, sera, and extracts of aortic intima on DEAE-Sephacel column. The purified 85-kD M-CSF (hatched bars) and PG-M-CSF (open bars) (a), sera from five normal volunteers $(b)$, extracts of normal intima obtained from normal aortic region of five patients with atherosclerosis $(c)$, and extracts of intima obtained from atherosclerotic region from the same individuals $(d)$ were loaded onto DEAE-Sephacel column. The bound materials were eluted through the column stepwisely with buffers containing increasing concentrations of $\mathrm{NaCl}$. The concentration of $\mathrm{NaCl}$ is indicated on the abscissa. The eluted fractions were assayed for M-CSF content, using an ELISA. The 85-kD M-CSF and PG-M-CSF were analyzed by SDS-PAGE, followed by immunoblotting ( $a$ inset).

\section{Discussion}

Evidence for the existence, in vivo, of the LDL-PG complex has been obtained in biochemical and histochemical studies of aortic lesions $(8,10)$. The in vitro interaction between LDL and PG extracted from aorta has also been demonstrated in numerous studies (3-7). However, many other aspects of this important interaction remain to be investigated. For example, Vijayagopal et al. (4) found that the binding of free GAG chains to LDL was weaker than that of the parental PG, while Mourão et al. (5) showed that removal of the core protein of aortic PG did not affect the interaction of the PG with LDL. The mechanism whereby macrophage uptake of the LDL-PG complex occurs, however, is not clear; one group reported that LDL modified by PG was metabolized via apolipoprotein B receptors (16), while another reported that the complex was metabolized via a scavenger-receptor pathway (25). These discrepancies could be due to differences in assay conditions or PG preparations. Most of these studies were done with unfractionated or partially fractionated PG extracted from the aorta. In our study, we clearly showed the affinity of PG-M-CSF and LDL (Figs. 1 and 2). To our knowledge, this study represents the first characterization of a specific proteoglycan molecule involved in the binding of LDL. It is possible that the apparent reduction in molecular size of PG-M-CSF preincubated with LDL (Fig. $1 b$ ) is due to structural alterations in the linear GAG chain induced by LDL binding, since the interaction between PGM-CSF and LDL is mediated by the binding of PG-M-CSF chondroitin sulfate GAG chains to LDL (Fig. 2, $a$ and $b$ ). However, the underlying mechanism in this phenomenon remains to be determined. In any case, the finding supports the conclusion that PG-M-CSF and LDL interact and form a complex.

Earlier studies have demonstrated the importance of M-CSF in the atherosclerotic process. First, several recent studies have reported the elevated expression of the M-CSF gene in vascular cell components of atherosclerotic lesions, including endothelial cells, monocytes/macrophages, and smooth muscle cells (2629). Our findings presented here, i.e., the presence of PG-M$\mathrm{CSF}$ and the 85-kD M-CSF in the aortic tissue and the increased amounts of these M-CSF in atherosclerotic lesions (Fig. 3, c and $d$ ), are in accordance with these observations. Since little PG-M-CSF was present in serum (Fig. $3 b$ ), the increment of PG-M-CSF amount in atherosclerotic lesions might reflect the coaccumulation of PG-M-CSF with LDL. Second, it has been reported that the $85-\mathrm{kD}$ M-CSF stimulates lipoprotein lipase secretion from macrophages and that it enhances both the uptake and efflux of cholesterol in human monocyte-derived macrophages (30). Both PG-M-CSF and the 85-kD M-CSF act on monocytes, as described previously (22); thus, it can reasonably be assumed that PG-M-CSF modulates LDL metabolism in macrophages.

Although the biologically active nature of PG-M-CSF and its binding of LDL suggest that this molecule makes a profound contribution to the atherosclerotic process, this contribution is still speculative. However, our study offers a new opportunity to study the nature of the interaction between PG and LDL and the subsequent metabolism of such complexes in macrophages.

\section{Acknowledgment}

This work was supported in part by grants from the Ministry of Education, Science and Culture, Japan.

\section{References}

1. Ruoslahti, E., and Y. Yamaguchi. 1991. Proteoglycans as modulators of growth factor activities. Cell. 64:867-869. 
2. Kjellén, L., and U. Lindahl. 1991. Proteoglycans: structures and interactions. Annu. Rev. Biochem. 60:443-475.

3. Camejo, G., H. Acquatella, and F. Lalaguna. 1980. The interaction of low density lipoproteins with arterial proteoglycans: an additional risk factor? Atherosclerosis. 36:55-65.

4. Vijayagopal, P., S. R. Srinivasan, B. Radhakrishnamurthy, and G. S. Berenson. 1981. Interaction of serum lipoproteins and a proteoglycan from bovine aorta. J. Biol. Chem. 256:8234-8241.

5. Mourão, P. A. S., and C. A. Bracamonte. 1984. The binding of human aortic glycosaminoglycans and proteoglycans to plasma low density lipoproteins. Atherosclerosis. 50:133-146.

6. Owens, R. T., and W. D. Wagner. 1991. Proteoglycans produced by cholesterol-enriched macrophages bind plasma low density lipoprotein. Atherosclerosis. 91:229-240.

7. Christner, J. E., and J. R. Baker. 1990. A competitive assay of lipoprotein: proteoglycan interaction using a 96-well microtitration plate. Anal. Biochem. 184:388-394.

8. Steele, R. H., W. D. Wagner, H. A. Rowe, and I. J. Edwards. 1987. Artery wall derived proteoglycan-plasma lipoprotein interaction: lipoprotein binding properties of extracted proteoglycans. Atherosclerosis. 65:51-62.

9. Wegrowski, J., M. Moczar, and L. Robert. 1986. Proteoglycan from pig aorta: comparative study of their interactions with lipoproteins. Biochem. J. 235:823-831.

10. Srinivasan, S. R., B. Radhakrishanamurthy, E. R. Dalferes, Jr., and G. S. Berenson. 1979. Collagenase-solubilized glycosaminoglycan complex of human aortic fibrous plaque lesions. Atherosclerosis. 34:105-118.

11. Toledo, O. M. S., and P. A. S. Mourão. 1980. Sulfated glycosaminoglycans in normal aortic wall of different mammals. Artery. 6:341-353.

12. Alavi, M., and S. Moore. 1985. Glycosaminoglycan composition and biosynthesis in the endothelium covered neointima of deendothelialized rabbit aorta. Exp. Mol. Pathol. 42:389-400.

13. Salisbury, G. B. J., D. P. Hajjar, and C. R. Minick. 1985. Altered glycosaminoglycan metabolism in injured arterial wall. Exp. Mol. Pathol. 42:306-319.

14. Hoff, H. F., and W. D. Wagner. 1986. Plasma low density lipoprotein accumulation in the aortas of hypercholesterolemic swine correlates with modifcations in aortic glycosaminoglycan composition. Atherosclerosis. 61:231-236.

15. Salisbury, G. B. J., D. J. Falcone, and C. R. Minick. 1985. Insoluble low density lipoprotein-proteoglycan complexes enhance cholesteryl ester accumulation in macrophages. Am. J. Pathol. 120:6-11.

16. Hurt-Camejo, E., G. Camejo, B. Rosengren, O. Wiklund, and G. Bondjers. 1990. Arterial proteoglycans increase the rate of oxidation of low density lipoproteins and its uptake by macrophages. Circulation. 82:III-559a.(Abstr.)

17. Clark, S. C., and R. Kamen. 1987. The human hematopoietic colonystimulating factors. Science (Wash. DC). 236:1229-1237.

18. Motoyoshi, K., F. Takaku, H. Mizoguchi, and Y. Miura. 1978. Purification and some properties of colony-stimulating factor from normal human urine. Blood. 52:1012-1020.

19. Wong, G. G., P. A. Temple, A. N. Leary, J. S. Witek-Giannotti, Y.-C. Yang, A. B. Ciarletta, M. C. Chung, P. Murtha, R. Kriz, R. J. Kaufman, et al. 1987. Human CSF-1: molecular cloning and expression of 4-Kb cDNA encoding the human urinary protein. Science (Wash. DC). 235:1504-1508.

20. Price, L. K. H., H. U. Choi, L. Rosenberg, and E. R. Stanley. 1992. The predominant form of secreted colony-stimulating factor- 1 is a proteoglycan. $J$. Biol. Chem. 267:2190-2199.

21. Suzu, S., T. Ohtsuki, N. Yanai, Z. Takatsu, T. Kawashima, F. Takaku, N. Nagata, and K. Motoyoshi. 1992. Identification of a high molecular weight macrophage colony-stimulating factor as a glycosaminoglycan-containing species. J. Biol. Chem. 267:4345-4348.

22. Suzu, S., T. Ohtsuki, M. Makishima, N. Yanai, T. Kawashima, N. Nagata, and K. Motoyoshi. 1992. Biological activity of a proteoglycan form of macrophage colony-stimulating factor and its binding to type V collagen. J. Biol. Chem. 267:16812-16815.

23. Hara, I., and M. Okazaki. 1986. High-performance liquid chromatography of serum lipoproteins. Methods Enzymol. 129:57-78.

24. Suzu, S., N. Yanai, Y. Sato-Somoto, M. Yamada, T. Kawashima, T. Hanamura, N. Nagata, F. Takaku, and K. Motoyoshi. 1991. Characterization of macrophage colony-stimulating factor in body fluids by immunoblot analysis. Blood. 77:2160-2165.

25. Vijayagopal, P., S. R. Srinivasan, B. Radhakrishnamurthy, and G. S. Berenson. 1993. Human monocyte-derived macrophages bind low-density-lipoprotein-proteoglycan complexes by a receptor different from the low-densitylipoprotein receptor. Biochem. J. 289:837-844.

26. Rajavashisth, T. B., A. Andalibi, M. C. Territo, J. A. Berliner, M. Navab, A. M. Fogelman, and A. J. Lusis. 1990. Induction of endothelial cell expression of granulocyte and macrophage colony-stimulating factors by modified low-density lipoproteins. Nature (Lond.). 344:254-257.

27. Rosenfeld, M. E., S. Ylä-Herttuala, B. A. Lipton, V. A. Ord, J. L. Lipton, and D. Steinberg. 1992. Macrophage colony-stimulating factor mRNA and protein in atherosclerotic lesions of rabbits and humans. Am. J. Pathol. 140:291-300.

28. Clinton, S. K., R. Underwood, L. Hayes, M. L. Sherman, D. W. Kufe, and P. Libby. 1992. Macrophage colony-stimulating factor gene expression in vascular cells and in experimental and human atherosclerosis. Am. J. Pathol. $140: 301-316$

29. Shimada, M., T. Inaba, H. Shimano, T. Gotoda, Y. Watanabe, K. Yamamoto, K. Motoyoshi, Y. Yazaki, and N. Yamada. 1992. Platelet-derived growth factor BB-dimer suppresses the expression of macrophage colony-stimulating factor in human vascular smooth muscle cells. J. Biol. Chem. 267:15455-15458

30. Ishibashi, S., T. Inaba, H. Shimano, K. Harada, I. Inoue, H. Mokuno, N Mori, T. Gotoda, F. Takaku, and N. Yamada. 1990. Monocyte colony-stimulating factor enhances uptake and degradation of acetylated low density lipoprotein and cholesterol esterification in human monocyte-derived macrophages. J. Biol. Chem. 265:14109-14117. 